\title{
CAPACIDAD DE LOS PACIENTES PARA TOMAR DECISIONES EN SALUD. ACTITUD Y SIGNIFICADO PARA MÉDICOS Y ABOGADOS
}

\author{
Gladys Bórquez Estefo*, Nina Horwitz Campos**, Ilse López Bravo*** y \\ Gina Raineri Bernain****
}

\begin{abstract}
Resumen: El objetivo es examinar las actitudes de un grupo de médicos y abogados que ejercen en Santiago de Chile hacia la aplicación de un protocolo que evalúa la capacidad de los pacientes para tomar decisiones autónomas en salud. Las respuestas se agruparon en cuatro dimensiones: valoración, factibilidad, aceptación y utilidad. Se aplicó la prueba de Wilocoxon rank-sum para evaluar la significación de la distribución de las diferencias valorativas. Los resultados revelan una valoración globalmente positiva de abogados y médicos respecto de la pertinencia y utilidad de estandarizar los procedimientos. Los médicos privilegian los aspectos prácticos y los abogados el resguardo de la autonomía de los pacientes y los derechos de las personas. La consideración de la opinión de los actores médicos y abogados resulta indispensable cuando una bioética cívica se fundamenta en la ética dialógica.
\end{abstract}

Palabras clave: capacidad, competencia, consentimiento informado, actitud, valores, diferencial semántico, investigación cualitativa, derechos humanos

\section{THE CAPACITY OF PATIENTS TO MAKE DECISIONS IN HEALTH: ATTITUDE AND MEANING FOR PHYSICIANS AND LAWYERS}

\begin{abstract}
The objective of this article is to examine the attitudes of a group of physicians and lawyers that practice in Santiago, Chile toward the application of a protocol which evaluates the capacity of patients to make autonomous health decisions. The responses were grouped in four dimensions: assessment, feasibility, acceptance and usefulness. The Wilocoxon rank-sum test was applied to evaluate the distribution significance of value differences. The results revealed a globally positive assessment of lawyers and physicians to the pertinence and usefulness of standardization of procedures. The physicians emphasized the practical aspects and the lawyers, protection of patient autonomy and human rights. Consideration of the opinions of medical and legal actors is indispensable when a civic bioethics is founded in ethical dialogue.
\end{abstract}

Key words: capacity, competence, informed consent, attitude, values, differential semantics, qualitative investigation, human rights

\section{CAPACIDADE DOS PACIENTES PARA TOMAR DECISÓES EM SAÚDE. ATITUDE E SIGNIFICADO PARA MÉDICOS E ADVOGADOS}

Resumo: O objetivo é examinar as atitudes de um grupo de médicos e advogados que atuam em Santiago do Chile sobre a aplicação de um protocolo que avalia a capacidade dos pacientes para tomar decisôes autônomas em saúde. As respostas foram agrupadas em quatro dimensôes: valoração, factibilidade, aceitação e utilidade. Aplicou-se a prova de Wilocoxon rank-sum para avaliar a significação da distribuição das diferenças valorativas. Os resultados revelam uma valoração globalmente positiva de advogados e médicos com respeito à pertinência e utilidade de padronizar os procedimentos. Os médicos privilegiam os aspectos práticos e os advogados o resguardo da autonomia dos pacientes e os direitos das pessoas. A consideraçáo da opinião dos autores médicos e advogados resulta indispensável quando uma bioética cívica se fundamenta na ética dialógica.

Palavras-chave: capacidade, competência, consentimento informado, atitude, valores, diferencial semântico, pesquisa qualitativa, direitos humanos

\footnotetext{
Médico. Profesora Departamento de Bioética y Humanidades Médicas, Facultad de Medicina, Universidad de Chile, Chile

** Socióloga. Profesora Departamento de Bioética y Humanidades Médicas, Facultad de Medicina Universidad de Chile, Chile

*** Licenciada en Estadísticas de Salud. Profesora Departamento Educación en Ciencias de la Salud, Facultad de Medicina, Universidad de Chile, Chile

**** Abogado y Enfermera. Profesora Departamento de Bioética y Humanidades Médicas, Facultad de Medicina, Universidad de Chile, Chile Correspondencia: gborquez@chp.cl
} 


\section{Introducción}

La existencia de una relación diferente entre el enfermo y el profesional de la salud, que enfatiza la deliberación y participación conjunta, se traduce en el proceso de consentimiento informado. Su esencia es considerar que las decisiones en salud, para ser moralmente aceptables, deben ser autónomas. En salud una decisión es autónoma cuando cumple tres condiciones: voluntariedad, información y capacidad.

En una publicación anterior(1) realizamos una extensa revisión de los conceptos de capacidad sanitaria y capacidad legal para la toma de decisiones, su evaluación y las propuestas para realizarla, las normativas legales y sanitarias vigentes en Chile, y los problemas más importantes en la práctica diaria que derivan de la comprensión, uso y evaluación de la capacidad.

En nuestro medio, la deliberación en torno a los principios básicos de una bioética plural es aún incipiente. Para responder a la interrogante sobre la valoración y las actitudes hacia la autonomía y la capacidad de las personas por parte de quienes evalúan esa capacidad, se optó por una investigación cualitativa con dos componentes. Una parte, cuyos resultados ya publicamos, indagó sobre las prácticas habituales de médicos y abogados en la evaluación de la capacidad para tomar decisiones en salud en nuestro medio y las nociones que las fundamentan(2). Observamos que éstas estaban, en general, asociadas a sus respectivos marcos epistémicos disciplinarios y, en particular, al contexto específico y las contingencias de su práctica.

Las dificultades percibidas dicen relación con aspectos prácticos, como la falta de un instrumento de medición que asegure su fácil aplicabilidad y que obtenga aceptación general y comprensibilidad de médicos, abogados y jueces. La relevancia que tiene la evaluación de la capacidad para la práctica profesional de médicos y abogados es alta, aunque por razones diferentes.

Los médicos que ejercen en geriatría y adolescencia tienen más claridad de la vinculación entre el consentimiento informado y el ejercicio de derechos de ciudadanía fundamentales, es decir, que del resultado de la evaluación de la capacidad de un paciente para tomar decisiones en salud le puede quedar vedada la gestión del propio cuerpo, siendo entregada a otras personas que la sociedad considera, hasta ahora, "especialmente calificadas para ello": médicos, gobernantes, sacerdotes y jueces, lo cual habla del énfasis ético que tradicio- nalmente se ha puesto en el principio de beneficencia en la relación médico-paciente. En cambio, es crucial para los abogados porque, al tratarse de la capacidad de obrar, define tanto la validez de determinados actos civiles como la responsabilidad en casos jurídicos, tanto civiles como penales.

En esta oportunidad expondremos los resultados de la segunda parte del estudio, cuyo objetivo es examinar las actitudes y significados asociados a un objeto que, en este caso, se refiere a "los procedimientos para evaluar la capacidad". Con este fin se aplicó una escala de diferencial semántico.

\section{Metodología}

\subsection{Acerca del instrumento de recolección de información}

La escala de diferencial semántico aplicada en este trabajo es una técnica que mide el significado de un objeto para un individuo. Esta técnica se originó en los trabajos de Charles Osgood, en la década de los cincuenta, como una forma de ubicar a los sujetos de acuerdo con sus reacciones ante pares de adjetivos bipolares en relación con determinados objetos o conceptos(3). Se le solicita al entrevistado que clasifique un objeto (real o conceptual) según una serie de miniescalas bipolares que describen aspectos de interés de éste. El supuesto teórico subyacente es que las respuestas del sujeto reflejarían su actitud cuando las dimensiones sobre las cuales se indaga generan valores diferenciales.

Una palabra o una frase corta constituyen el constructo de interés u objeto. Los sujetos, por su parte, contribuyen a describir su significado respondiendo a un conjunto de pares de adjetivos bipolares a los cuales se les adjudica un puntaje dentro de un continuo, por ejemplo, entre $(+\mathrm{X})$ a $(-\mathrm{X})$ o entre $\mathrm{X}$ a $(\mathrm{X}+\mathrm{Y})$, en el estilo de la escala Lickert.

El diferencial semántico puede usarse para examinar diferencias entre distintos grupos o individuos sobre el significado de un concepto, y entre diferentes conceptos para el mismo grupo o individuo. Se ha demostrado que, independiente de la forma en que se determinen los aspectos del objeto, habitualmente se generan tres grandes categorías referidas a dimensiones actitudinales básicas del objeto o concepto en cuestión: la valoración integral positiva o negativa; la percepción de su fuerza o potencia, y la percepción de su actividad o acción(3). 


\subsection{Elaboración del instrumento}

En este caso, la escala de diferencial semántico formó parte de una entrevista semiestructurada dirigida a médicos y abogados. La entrevista tuvo por fin capturar el manejo conceptual explícito e implícito de ambos grupos profesionales en relación con la autonomía de los pacientes para tomar decisiones que atañen a su salud. La información recogida a través de la entrevista fue analizada cualitativamente y ha sido objeto de una publicación anterior(2).

El diferencial semántico incorporado a la mencionada entrevista ha sido analizado separadamente, tanto por motivos conceptuales como metodológicos. Este instrumento pretende informar de los aspectos menos declarativos de los sujetos y más vinculados con juicios valorativos, sensaciones y/o emociones evocadas por las temáticas tratadas en la entrevista. Por otra parte, pese a estar ligado epistemológicamente a un marco cualitativo de investigación, puede ser sometido a análisis cuantitativo.

Los instrumentos de diferencial semántico elaborados para médicos y abogados tuvieron algunas diferencias, buscando ajustarse a las circunstancias y características más pertinentes y relevantes para cada escenario de práctica. El supuesto que lo sustenta es que las respuestas de los sujetos, como ya se afirmó, se acercarían a la actitud que ya tienen configurada sobre el objeto indagado.

El proceso se inició creando una serie de 23 adjetivos polares que cubrieran el campo de la discusión que generaría entre los médicos la posibilidad de "estandarizar los procedimientos que evalúan la capacidad del paciente para tomar sus decisiones en materia de salud" (objeto a evaluar).

Luego, se aplicó una prueba piloto a un grupo de 17 médicos para examinar la pertinencia conceptual del enunciado y del conjunto de pares de adjetivos polares descriptivos. Como resultado de la consulta, se decidió reformular el enunciado (u objeto) de la cédula de entrevista reemplazándolo por la opinión del sujeto frente a "utilizar una guía o protocolo consensuado para evaluar la capacidad de los pacientes".

Se sometió nuevamente a escrutinio la escala entre un grupo de médicos y abogados (31 en cada caso). Con estos resultados, se realizó un análisis descriptivo univariado para identificar aquellos ítems o factores que presentaran un comportamiento poco definido -es decir, sobre los cuáles los sujetos en su conjunto no se pronunciaran por ninguno de los dos polos-con el fin de eliminarlos.

A continuación, se aplicó un modelo multifactorial para detectar ítems que estuvieran midiendo las mismas dimensiones. De este modo, la escala dirigida a los médicos quedó compuesta finalmente de 16 ítems y la de abogados con 20 .

Los ítems de ambas escalas se agrupan en las siguientes cuatro dimensiones teóricas:

a) Valoración, definida como la acción de reconocer, estimar o apreciar el valor o mérito de utilizar una guía o protocolo consensuado para evaluar la capacidad de los pacientes en materia de salud.

b) Factibilidad, entendida como el conjunto de condiciones que determina que una guía o protocolo de la naturaleza descrita sea posible de realizar o de aplicar.

c) Aceptación o acción íntima y subjetiva de recibir voluntariamente y sin oposición tal instrumento.

d) Utilidad, referida a las condiciones que definirían una eventual guía o protocolo como provechosa, conveniente y fructífera para la práctica profesional.

\subsection{Grupo de estudio}

La muestra estudiada estuvo compuesta por un total de 27 profesionales, 14 médicos y 13 abogados que ejercen en la Región Metropolitana de Santiago de Chile. El muestreo, que forma parte del diseńo cualitativo global del trabajo(2), no es aleatorio sino teórico o conceptual, en tanto su propósito es incluir a quienes mejor responden las preguntas del estudio. Se estimó que el punto de saturación de la información se alcanzaba con un total de 27 sujetos seleccionados en calidad de informantes clave.

\subsection{Análisis de datos}

El análisis de los datos siguió la siguiente secuencia:

1. Considerando los puntajes mínimos y máximos para cada aseveración del diferencial semántico, se calculó la mediana como medida de posición, porque se trataba de una escala discreta de puntajes en la que el sujeto asignaba un valor a cada una de las aseveraciones planteadas en el instrumento. 
2. Los resultados anteriores se presentaron separadamente para ambos grupos de profesionales, dado que las preguntas mostraban diferencias entre sí.

3. Se calculó la mediana de los puntajes, ahora agrupados por las cuatro dimensiones (valoración, factibilidad, aceptación y utilidad).
4. Se compararon los datos en aquellos ítems en que son comparables.

5. Se aplicó una prueba no paramétrica para dos muestras independientes (Wilocoxon rank-sum), con el fin de evaluar la significación de la distribución de las diferencias valorativas entre médicos y abogados estudiados.

\section{Resultados}

Tabla 1. Características de la muestra

\begin{tabular}{|l|l|c|}
\hline Profesión & Especialidad & $\mathrm{N}$ \\
\hline \multirow{4}{*}{ Médicos (14) } & Psiquiatría Adulto & 4 \\
\cline { 2 - 3 } & Psiquiatría Infantil & 2 \\
\cline { 2 - 3 } & Neurología Infantil & 3 \\
\cline { 2 - 3 } & Neurología Adulto & 1 \\
\cline { 2 - 3 } & Geriatría & 2 \\
\cline { 2 - 3 } & Adolescencia 1Pediatría/1Ginecología & 2 \\
\hline \multirow{5}{*}{ Abogados (13) } & Derecho Civil & 1 \\
\cline { 2 - 3 } & Derecho Penal & 2 \\
\cline { 2 - 3 } & Derecho Sanitario & 3 \\
\cline { 2 - 3 } & Derecho de Familia & 2 \\
\cline { 2 - 3 } & Juez(a) de Menores & 1 \\
\cline { 2 - 3 } & Ministro(a) Corte Apelaciones & 1 \\
\cline { 2 - 3 } & Derecho de Menores & 2 \\
\cline { 2 - 3 } & Notario Público & \\
\hline N (27) & & \\
\hline
\end{tabular}

El análisis por separado de las respuestas de abogados y médicos entrevistados ante el planteamiento de utilizar una guía o protocolo consensuado para evaluar la capacidad de los pacientes reveló una actitud más favorable de parte de los abogados que de los médicos. Como puede observarse en las medianas de puntaje de las tablas 2 a 5, a continuación, la proporción de puntajes favorables (cercanos o sobre la mediana) en el caso de los abogados es del 50\% (tabla 2) y en el de los médicos alcanza el 31\% (tabla 4). En el caso de estos últimos, además, las respuestas tienden a concentrarse en un menor número de ítems.

Tabla 2. Puntaje obtenido por 14 abogados

\begin{tabular}{|l|c|c|c|}
\hline Ítems & Mediana & Mínimo & Máximo \\
\hline Resguarda autonomía del paciente & 6.5 & 1 & 7 \\
\hline Ni eficaz ni ineficaz & 4 & 1 & 7 \\
\hline Útil para la práctica legal & 5.5 & 1 & 7 \\
\hline De interés para la práctica legal & 5 & 1 & 7 \\
\hline Impracticable en la realidad médico legal actual & 3.5 & 1 & 7 \\
\hline Protector del ejercicio de los derechos de las personas & 6 & 2 & 7 \\
\hline Demasiado complicada para implementarla & 3 & 1 & 7 \\
\hline Ni cuestiona ni respalda el ejercicio de la capacidad legal & 4 & 0 & 7 \\
\hline No facilita ni entorpece el trabajo profesional de los abogados & 4 & 1 & 7 \\
\hline Pertinente para evaluar la capacidad de los pacientes & 6.5 & 2 & 7 \\
\hline Resguarda la autonomía de las personas & 7 & 1 & 7 \\
\hline Ahorra tiempo valioso en pro de la salud del paciente & 5 & 1 & 7 \\
\hline Importante para el ejercicio profesional & 7 & 1 & 7 \\
\hline
\end{tabular}


Capacidad de los pacientes para tomar decisiones en salud - Gladys Bórquez, Nina Horwitz, Ilse López y Gina Raineri

\begin{tabular}{|l|c|c|c|}
\hline Posible & 5 & 4 & 7 \\
\hline Difícil establecer diferencias entre la capacidad profesional de los abogados & 3.5 & 1 & 7 \\
\hline De interés tanto en el ámbito sanitario como legal & 5.5 & 1 & 7 \\
\hline Haría más cómoda la práctica profesional & 5.5 & 1 & 7 \\
\hline Ni valiosa ni sin valor para la práctica profesional de los abogados & 4 & 1 & 7 \\
\hline Ventajosa para el paciente en materia legal & 5.5 & 1 & 7 \\
\hline Facilita la relación médico-paciente & 6 & 1 & 7 \\
\hline
\end{tabular}

Tabla 3. Distribución de ítems según mediana de puntaje (abogados)

\begin{tabular}{|l|c|c|}
\hline Mediana & No $^{\circ}$ & $\%$ \\
\hline 7 & 2 & 10 \\
6.5 & 2 & 10 \\
6 & 2 & 10 \\
5.5 & 4 & 20 \\
5 & 3 & 15 \\
4 & 4 & 20 \\
3,5 & 2 & 10 \\
3 & 1 & 5 \\
\hline TOTAL & 20 & 100 \\
\hline
\end{tabular}

Tabla 4. Puntaje obtenido por 17 médicos

\begin{tabular}{|l|c|c|c|}
\hline Ítems & Mediana & Mínimo & Máximo \\
\hline Eficaz & 6 & 1 & 7 \\
\hline Útil para la práctica médica & 5 & 1 & 7 \\
\hline No hace ni más personal ni más impersonal la relación médico-paciente & 4 & 1 & 7 \\
\hline No es ni practicable ni impracticable en la realidad del medio actual & 4 & 1 & 7 \\
\hline Protege el ejercicio profesional & 5 & 1 & 7 \\
\hline Ni simple ni complicada para aplicarla & 4 & 2 & 7 \\
\hline No cuestiona el ejercicio profesional & 6 & 1 & 7 \\
\hline Facilitador del trabajo profesional & 6 & 2 & 7 \\
\hline Pertinente para evaluar la capacidad de los pacientes & 6 & 1 & 7 \\
\hline No resguarda ni atenta contra la autonomía del paciente & 4 & 1 & 7 \\
\hline Ni necesaria ni innecesaria para la práctica profesional & 4 & 2 & 7 \\
\hline Importante & 5 & 2 & 7 \\
\hline Posible de aplicar & 6 & 3 & 7 \\
\hline Expone al médico en materias legales & 2 & 1 & 4 \\
\hline Potencia el juicio profesional de cada médico & 5 & 0 & 7 \\
\hline Una iniciativa valiosa para la práctica profesional & 5 & 3 & 7 \\
\hline
\end{tabular}

Tabla 5. Distribución de ítems según mediana de puntaje médicos

\begin{tabular}{|c|c|c|}
\hline Mediana & No $^{\circ}$ & $\%$ \\
\hline 6 & 5 & 31,3 \\
\hline 5 & 5 & 31,3 \\
\hline 4 & 5 & 31,3 \\
\hline 2 & 1 & 6,1 \\
\hline TOTAL & 16 & 100 \\
\hline
\end{tabular}

El gráfico 1 compara la mediana de los puntajes entre médicos y abogados en 12 ítems comunes de las escalas utilizadas para cada grupo. 
Gráfico 1. Mediana ítems médicos y abogados

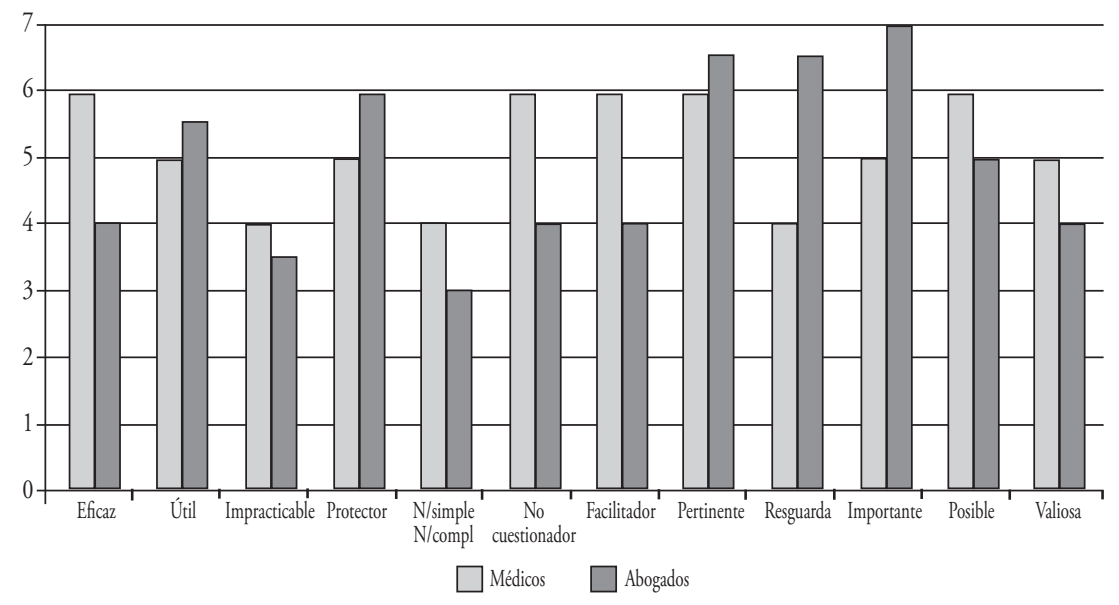

Se aprecia que ambos grupos coinciden en destacar su pertinencia y utilidad. Los médicos privilegian los aspectos prácticos de disponer de un protocolo común para evaluar la capacidad de los pacientes, estimándolo como eficaz, no cuestionador del trabajo profesional y facilitador de éste, y consideran que sería valioso y posible de ser aplicado. Por su parte, los abogados estimaron que sería importante para el ejercicio profesional, enfatizando el resguardo de la autonomía de los pacientes y de los derechos de las personas.
La mediana de los puntajes fue luego agrupada de acuerdo con las cuatro dimensiones ya descritas para las respuestas obtenidas: valoración, factibilidad, utilidad y aceptación de la idea de disponer de una guía o protocolo común. Las tablas 6 y 7, a continuación, presentan el orden de importancia asignado a las dimensiones por cada grupo profesional.

Tabla 6. Resultados por dimensión abogados

\begin{tabular}{|l|c|c|c|}
\hline Dimensión valoración & Mediana & Mínimo & Máximo \\
\hline 1. Resguarda la autonomía del paciente & 6.5 & 1 & 7 \\
\hline 4. Interesa para la práctica legal & 5 & 1 & 7 \\
\hline 11. Resguarda la autonomía de las personas & 7 & 1 & 7 \\
\hline 13. Importante para el ejercicio profesional & 7 & 1 & 7 \\
\hline 18. Ni valiosa ni sin valor para la práctica profesional de los abogados & 4 & 1 & 7 \\
\hline Mediana & 6.5 & & \\
\hline
\end{tabular}

\begin{tabular}{|l|c|c|c|}
\hline Dimensión aceptación & Mediana & Mínimo & Máximo \\
\hline & 6 & 2 & 7 \\
\hline 6. Protector del ejercicio de los derechos de las personas & 4 & 0 & 7 \\
\hline 8. Ni cuestiona ni respalda el ejercicio de la capacidad legal & 6.5 & 2 & 7 \\
\hline 10. Pertinente para evaluar la capacidad de los pacientes & 5.5 & 1 & 7 \\
\hline 16. De interés tanto en el ámbito sanitario como legal & 6 & 1 & 7 \\
\hline 20. Facilita la relación médico-paciente & 6 & & \\
\hline Mediana & & \\
\hline
\end{tabular}




\begin{tabular}{|l|c|c|c|}
\hline Dimensión utilidad & Mediana & Mínimo & Máximo \\
\hline & 5.5 & 1 & 7 \\
\hline 3. Útil para la práctica legal & 4 & 1 & 7 \\
\hline 9. Ni facilita ni entorpece el trabajo profesional de los abogados & 5 & 1 & 7 \\
\hline 12. Ahorra tiempo valioso en pro de la salud del paciente & 3.5 & 1 & 7 \\
\hline 15. Difícil establecer diferencias entre la capacidad profesional de los abogados & 5.5 & 1 & 7 \\
\hline 19. Ventajosa para el paciente en materia legal & 5 & & \\
\hline Mediana & & \\
\hline
\end{tabular}

\begin{tabular}{|l|c|c|c|}
\hline Dimensión factibilidad & Mediana & Mínimo & Máximo \\
\hline & 4 & 1 & 7 \\
\hline 2. Ni eficaz ni ineficaz & 3.5 & 1 & 7 \\
\hline 5. Impracticable en la realidad del médico legal actual & 3 & 1 & 7 \\
\hline 7. Complicada para implementarla & 5 & 4 & 7 \\
\hline 14. Posible & 5.5 & 1 & 7 \\
\hline 17. Haría más cómoda la práctica profesional & 4 & & \\
\hline Mediana & & \\
\hline
\end{tabular}

Tabla 7. Resultados por dimensión médicos

\begin{tabular}{|l|c|c|c|}
\hline Dimensión valoración & Mediana & Mínimo & Máximo \\
\hline & 4 & 1 & 7 \\
\hline 10. Resguarda la autonomía de los pacientes & 4 & 2 & 7 \\
\hline 11. Necesaria para la práctica profesional & 5 & 2 & 7 \\
\hline 12. Importante & 5 & 3 & 7 \\
\hline 16. Valiosa para la práctica profesional & 4.5 & & \\
\hline Mediana & & \\
\hline
\end{tabular}

\begin{tabular}{|l|c|c|c|}
\hline Dimensión factibilidad & Mediana & Mínimo & Máximo \\
\hline & 6 & 1 & 7 \\
\hline 1. Eficaz & 4 & 1 & 7 \\
\hline 4. Impracticable en la realidad del medio actual & 4 & 2 & 7 \\
\hline 6. Ni complicada ni fácil de aplicar & 6 & 3 & 7 \\
\hline 13. Posible & 5 & & \\
\hline Mediana & 5 \\
\hline
\end{tabular}

\begin{tabular}{|l|c|c|c|}
\hline Dimensión utilidad & Mediana & Mínimo & Máximo \\
\hline & 5 & 1 & 7 \\
\hline 2. Útil para la práctica médica & 6 & 2 & 7 \\
\hline 8. Facilita el trabajo profesional & 2 & 1 & 4 \\
\hline 14.Expone al médico en materias legales & 5 & 0 & 7 \\
\hline 15.Potencia el juicio profesional de cada médico & 5 & & \\
\hline Mediana & 5 \\
\hline
\end{tabular}

\begin{tabular}{|l|c|c|c|}
\hline Dimensión aceptación & Mediana & Mínimo & Máximo \\
\hline 3. Relación médico-paciente impersonal & 4 & 1 & 7 \\
\hline 5. Protector del ejercicio profesional & 5 & 1 & 7 \\
\hline 7. No cuestiona el ejercicio profesional & 6 & 1 & 7 \\
\hline 9. Pertinente para evaluar la capacidad de los pacientes & 6 & 1 & 7 \\
\hline Mediana & 5.5 & & \\
\hline
\end{tabular}


Para los abogados la dimensión valorativa es la más importante; para los médicos, en cambio, es la de aceptación, seguida por las de utilidad y factibilidad con iguales puntajes. Estas últimas consideraciones ocupan los lugares de menor importancia en las respuestas de los abogados.

Estas diferencias se muestran con mayor profundidad en los gráficos siguientes, que comparan las respuestas entre médicos y abogados al interior de cada una de las dimensiones.

Gráfico 2. Dimensión valoración.

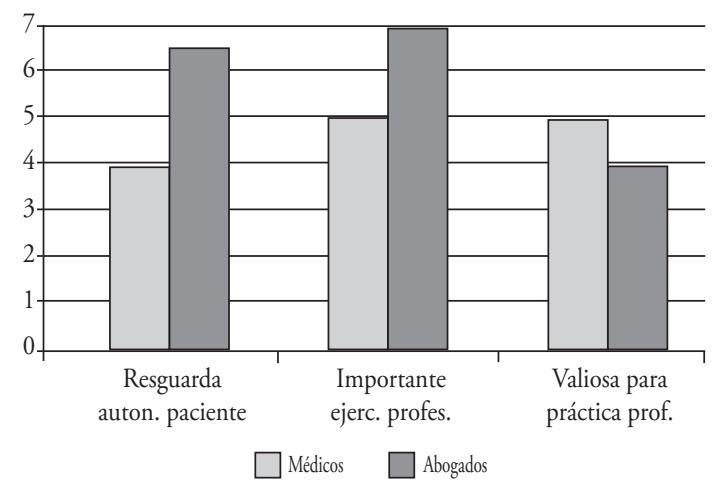

Los médicos valoran la importancia de un protocolo consensuado para el ejercicio profesional, mientras que los abogados el resguardo de la autonomía de los pacientes.

Gráfico 3. Dimensión factibilidad.

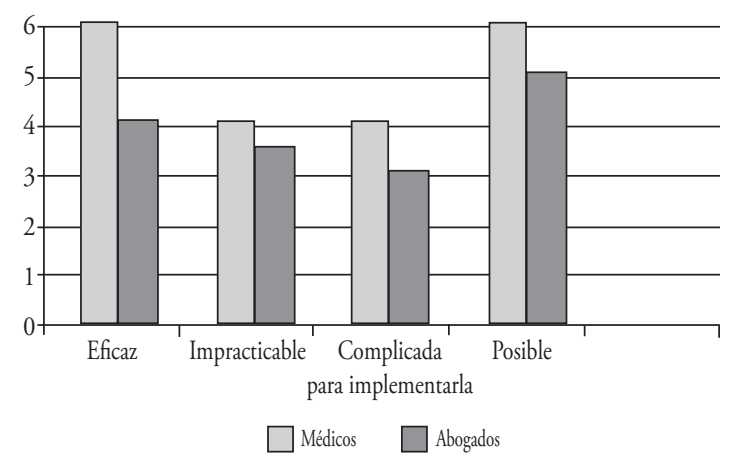

Las consideraciones de factibilidad parecen ser más centrales en las respuestas de los médicos que en las de los abogados, tomando en cuenta los primeros particularmente la alta eficacia y las condiciones de posibilidad de disponer de una guía común.
Gráfico 4. Dimensión utilidad.

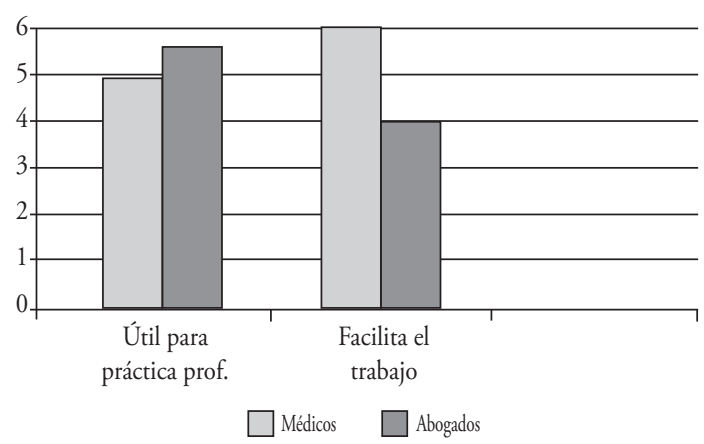

Gráfico 5. Dimensión aceptación.

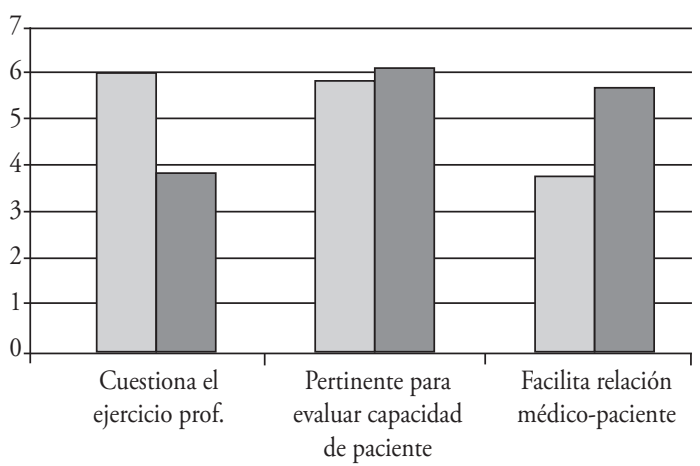

$\square$ Médicos $\square$ Abogados

Fundamentalmente, los médicos aceptarían un protocolo compartido porque lo consideran pertinente y porque no cuestionaría su ejercicio profesional, mientras los abogados lo aceptan también por su pertinencia y porque consideran que facilitaría la relación médico-paciente.

\section{Discusión}

El respeto por la autonomía de los pacientes constituye hoy una de las piedras angulares de la bioética, así como una aspiración fundamental para la práctica médica. La incorporación en la práctica clínica del consentimiento informado y de las normativas emanadas de los cuerpos legales que protegen los derechos de los pacientes en salud, obligan a plantearse el proceso de evaluación de la capacidad sanitaria para la toma de decisiones en la práctica clínica diaria.

El proceso que determina la capacidad de los pacientes para ejercer su autonomía no sólo es complejo sino que variado e inconsistente. Esto puede explicarse por diversos motivos. Uno es que el respeto por la autonomía como obligación ética debe a menudo armonizarse 
con otros principios que compiten, como prevenir el daño, actuar en función del beneficio de los pacientes y los problemas de asignación de recursos justos(4). Otro motivo es la ausencia de guías o protocolos de uso consensuado para estos efectos, lo que hace que el proceso de evaluación descanse en la experiencia y criterios propios de la práctica individual(2). Un tercer aspecto se relaciona con las actitudes de los profesionales encargados de evaluar la autonomía de los pacientes. Este es el foco del presente artículo.

En general, los resultados muestran que los abogados parecen tener una actitud más favorable hacia la posibilidad de contar con un protocolo o guía común para evaluar la capacidad sanitaria de los pacientes, lo cual nos parece explicable, dado que, para ellos, se trata de una tarea entregada a peritos especialistas, quienes contarían con las herramientas apropiadas. Por otra parte, la percepción y evaluación de los médicos nace desde las complejidades de la experiencia clínica.

La agrupación de las respuestas según las cuatro dimensiones que contempla el instrumento -valoración, factibilidad, utilidad y aceptación- corrobora esta apreciación, en cuanto el abogado da más importancia a la dimensión valorativa del objeto como un bien externo que resguarda la autonomía del paciente y los derechos de las personas. En cambio el médico prioriza el bien interno en la medida en que un instrumento estandarizado es posible y eficaz para medir, y útil para el ejercicio profesional. En tal sentido se corrobora la observación que hiciéramos cuando estudiamos las nociones de capacidad de los médicos y abogados, las que aparecen esencialmente determinadas por un contexto específico que proviene de sus respectivos marcos epistémicos disciplinarios(2).

En cualquier caso, la valoración globalmente positiva que hacen tanto abogados como médicos respecto de la pertinencia y utilidad de estandarizar los procedimientos que evalúan la capacidad del paciente para tomar sus decisiones en materia de salud es prometedora, si consideramos las consecuencias personales, sociales, morales y legales que conlleva declarar a un paciente "incapaz", con la consecuente pérdida de la capacidad de ejercicio de sus derechos. En virtud de ello, se hace imperioso contar con protocolos o herramientas para efectuar una adecuada evaluación de la capacidad de las personas, que aseguren no incurrir en una violación de sus derechos fundamentales.
La prioridad otorgada por los médicos a criterios de factibilidad, utilidad y aceptación para juzgar un protocolo consensuado pone de relieve no solamente la variada experiencia clínica sino también la responsabilidad legal de este grupo profesional al juzgar la capacidad de los pacientes. Para que la disponibilidad de medios probados y especializados de evaluación de la capacidad sea relevante para la práctica médica es necesario, por tanto, que su eventual implementación sea de amplia aceptación, lo que podría favorecer el proceso de consentimiento informado y el mayor respeto de los derechos del paciente.

La valoración más incondicional por un protocolo que revelan los abogados responde, a su vez, a la necesidad de disponer de un dato más preciso y confiable para los procesos de su propia incumbencia profesional. La dimensión valorativa que resaltan los abogados es promisoria además a la luz del actual proyecto de ley que se discute en el Parlamento chileno referente a los derechos y deberes de las personas en salud.

Aunque el modelo de relación clínica en nuestro medio sea aún de importante tradición paternalista y el diálogo bioético plural sea incipiente, encontramos un terreno propicio para entregar en el futuro a la comunidad médica una herramienta de evaluación más eficaz y justa que disminuya la variabilidad de una práctica médica intuitiva como la actual.

Considerar la opinión de los actores médicos y abogados resulta indispensable, en cuanto una bioética cívica se funda sobre el reconocimiento recíproco de la autonomía de cada ser racional, una autonomía personal vista como autorrealización individual, en la que existe tanto una concepción propia del bien como una intersubjetiva de lo correcto. La ética del discurso, en este sentido, se esfuerza por recuperar la intersubjetividad perdida durante la modernidad y la desaparición de la solidaridad entre los sujetos. El carácter dialógico de la razón humana, en condiciones ideales de simetría que incluya a todos los afectados, parece respetar tanto la pluralidad de visiones del bien como los mínimos universales.

Agradecemos a Ignacio Valenzuela y a Domingo Vallejos por su colaboración en la elaboración del instrumento y el trabajo de campo. 


\section{Referencias}

1. Bórquez G, Raineri G, Bravo M. La evaluación de la "capacidad de la persona”: en la práctica actual y en el contexto del consentimiento informado. Rev Med Chile 2004; 132: 1243-1248.

2. Bórquez G, Raineri G, Horwitz N, Huepe G. La noción de capacidad de la persona para tomar decisiones, en la práctica médica y legal. Rev Méd Chile 2007; 135: 1153-1159.

3. Osgood ChE. The Nature and Measurement of Meaning. Psychological Bulletin 1952; 49: 204-210.

4. Rogers WA. Whose autonomy? Which choice? A study of GPs' attitudes towards patient autonomy in the management of low back pain. Family Practice 2002; 19(2): 140-145.

Recibido: 18 de junio de 2008

Aceptado: 22 de agosto de 2008 